\title{
The relevance of the Lewy Body to the pathogenesis of idiopathic Parkinson's disease
}

\section{Accuracy of clinical diagnosis of idiopathic Parkinson's disease Andrew John Lees}

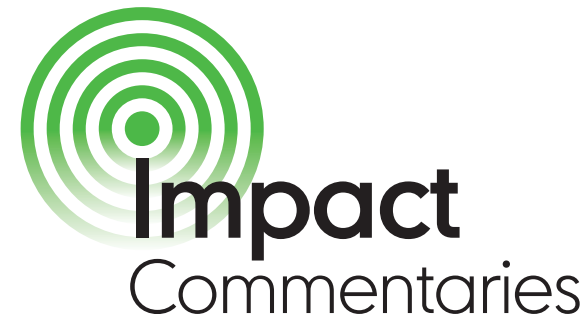

\begin{abstract}
Professor Andrew Lees of the National Hospital for Neurology and Neurosurgery, Queen Square describes the clinico-pathological studies at the Queen Square Brain Bank that have led to improved diagnostic accuracy of Parkinson's syndromes in neurological practice
\end{abstract}

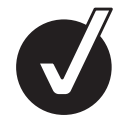

During my undergraduate training at the London Hospital, Whitechapel, the EDITOR'S teaching autopsy was an CHOICE eagerly anticipated ritual. The whole medical firm would troop across the road from the hospital to the mortuary where a clinician, often the consultant, would present the history and physical signs of the deceased and then the morbid anatomists would reveal the macroscopic pathological findings. Henry Urich perched on a ledge next to the cadaver would, with his exotic Eastern European accent, lead the discussion on all the neurological cases while we remained transfixed looking down in awe from the gallery. The naked eye appearances of the sliced brain allowed him to suspect diagnoses such as Parkinson's disease and exposed other pathologies, such as tumours and haemorrhage. These demonstrations taught me the rudiments of the classical anatomo-clinical neurological method, the great level of uncertainty relating to the cause of death that existed in so many cases, the frequency of

Correspondence to Professor A J Lees, Reta Lila Weston Institute of Neurological Studies, Institute of Neurology, University College London, London WC1N 1PJ, UK; andrew.lees@ucl.ac.uk
Title: THE RELEVANCE OF THE LEWY BODY TO THE PATHOGENESIS OF IDIOPATHIC PARKINSON'S DISEASE Authors: W R G Gibb, A J Lees Published: 1988;51:745-52

Title: ACCURACY OF CLINICAL DIAGNOSIS OF IDIOPATHIC PARKINSON'S DISEASE: A CLINICO-PATHOLOGICAL STUDY OF 100 CASES

Authors: Andrew J Hughes, Susan E Daniel, Linda Kilford, Andrew J Lees Published: 1992;55:181-4

multiple pathologies in an individual case and most of all the need for humility. A decade later autopsy rates began to tumble in the UK and the teaching autopsy was on its last legs; but for a few dissenting voices, nobody seemed to care. The opportunities to do careful clinicopathological studies were dwindling fast as neurochemistry and imaging took centre stage as neurological research priorities.

Shortly after I had become a consultant at University College Hospital, London, and Queen Square, I was flying to a meeting on Parkinson's disease in Vienna and had the good fortune to sit next to the late Professor David Marsden. Over a couple of drinks I told him that I wanted to set up a brain bank dedicated to Parkinson's disease and that the pathologist at Maida Vale Hospital, Dr Robin Barnard, was supportive of the idea. David, who had carried out important anatomical studies on the substantia nigra in animals as a medical student, was enthusiastic and on our return we obtained a 5 year programme grant from the Parkinson's Disease Society of the UK and the UK Parkinson's Brain Bank was born. The first frozen half brains were dispatched to the Institute of Psychiatry for neurochemical studies in Peter Jenner's laboratory while Bill Gibb, a MRC funded research fellow, and I worked on the formalin fixed tissue under Dr Barnard's direction at Maida Vale Hospital.

Bill and I started to review together the surprisingly limited and largely conflicting literature on the neuropathological findings in Parkinson's disease. Early revelations were that the substantia nigra lesion, first described in 1917 in the doctoral thesis of Konstantin Tretiakoff, a young Russian émigré working in Pierre Marie's laboratory, did not gain general acceptance for another 40 years and much more remarkably that the characteristic macroscopic bleaching of the midbrain, although described in postencephalitic Parkinsonism, was also not recognised in Parkinson's disease until the mid-1950s. The clinical data provided in the best histopathological reports were skimpy at best and the natural history in each case ignored.

Based on the available published data and expert opinion, we constructed tentative clinical operational criteria to use in a retrospective review of the available case notes of the early brain bank material to try to determine if we could predict whether an individual brain was likely to have severe loss of nigral pars compacta cells with brain stem Lewy bodies. Although our occasional review paper published in 1988 in JNNP focused primarily on the importance and relative selectivity of the Lewy Body for Parkinson's disease and the notion that individuals with incidental Lewy Body pathology might have been at greater risk of developing Parkinson's disease in life than age matched controls without Lewy bodies, this landmark paper has been mainly remembered and quoted for the first reference to the Queen Square Brain Bank criteria (the full details of which were published a year later in a little quoted paper: Gibb WRG, Lees AJ. Neuropath and Applied Neurobiology 1989;15:27-44). In this study of 269 patients generously provided from the 
collections of several British neuropathologists with a clinical diagnosis of parkinsonism, 78 were selected on the basis of the proposed operational criteria and 73 of these were found to fulfil accepted pathological criteria (severe nigral cell loss and brainstem Lewy bodies). This $6 \%$ mismatch was the start of the diagnostic accuracy work, which would follow. In devising the criteria, our aim was always to construct positive criteria from a basic starting point of exclusion criteria and this preliminary correlative work led to the proposal of prospective diagnostic criteria (step 3 OSBB criteria), that included unilateral onset, coarse pill rolling rest tremor, persistent limb asymmetry of signs, excellent response to L-dopa lasting more than 5 years and a disease duration of more than 10 years. ${ }^{1}$ The criteria were simple to use and started from a clinical perspective. They contrast strikingly with the detailed and frequently unwieldy consensus diagnostic criteria that are now so much in vogue in neurology and psychiatry. They were not intended for routine clinical use but we hoped that they might prove helpful for clinicopathological correlative research. The fact that they have been widely adopted and used in research projects all over the world for more than 20 years is both ironic and a pleasant surprise. The clamour for revised more inclusive criteria is now mounting as the diagnosis of Parkinson's disease especially in the first years of disease remains difficult. Let us hope that whichever panel of experts is delegated for the task will have wide clinical experience of the malady and take account of the heterogeneity of both the clinical phenomenena and disease course.

Andrew Hughes, a neurologist from Melbourne, then came to work at the Middlesex Hospital with Gerald Stern and me, and after he had got to know all about apomorphine therapy I set him a 'little side project' at the Queen Square Brain Bank. The idea was simply to compare the final clinical diagnosis of Parkinson's disease with the actual brain bank pathological diagnosis. Andrew later admitted to me that he was sceptical that such an elementary audit would produce a publication. Much of the work was done in the evening by Andrew working alone in the brain bank where he followed sometimes late into the night the course of a patient's life from the general practitioner case notes describing childhood illnesses and later to the first subtle emerging features of neurological disease and finally in the hospital case notes, the long increasingly emotional journey of Parkinson's disease. The project was straightforward and although the 'final' diagnosis of the first hundred brains did require a detailed review of the clinical notes rather than accepting what was recorded in the brain bank file, within a month Andrew had come up with a figure of $76 \%$. Twenty-four of the patients considered by neurologists to have Parkinson's disease at the time of death did not have the histopathological changes in the brain generally recognised to be associated with Parkinson's disease. When after clinic Andrew reported the figures to Gerald and me in our weekly research meeting on Thursday evening, I could hardly contain my excitement.

The orientation of the brain bank with the emphasis on the clinical findings of the donor in life made it a unique resource. 'The gift that keeps on giving' later proved to be the ongoing referencing of our paper by clinical researchers. During the study, Andrew was acutely aware of the enormous privilege it was to have access to the personal details of patients who had so generously donated their brains, and by piecing together their medical history from the notes at times it almost felt like some of them were coming back to life.

The 1992 paper describing the disappointing diagnostic accuracy for Parkinson's disease was sent to JNNP ('the green rag') as our first choice and accepted with minor revisions. When Andrew first presented the data at the Association of British Neurologists meeting in London, he received a frosty and intimidating questioning from several eminent senior neurologists. Surely this level of inaccuracy referred to general practitioners not neurologists? In North America and parts of Continental Europe, the poor figures were blamed on the relative dearth of neurologists in the UK and poor training. Perhaps fortunately for the longstanding success of our paper, a well respected movement disorder specialist Ali Rajput simultaneously reported in the Canadian Journal of Neurological Sciences identical figures from his own practice. ${ }^{2}$

Our study looked at clinical variables, which might improve the diagnosis of Parkinson's disease, including a retrospective application of the Queen Square Brain Bank criteria to the case notes and exposed the shortcomings of too stringent criteria, which only slightly reduced the misdiagnosis rate at the expense of excluding large numbers of true Parkinson's disease cases. The natural extension of this study was to return to the files and collect as much clinical information as possible on all 100 patients and examine whether retrospectively applying established diagnostic criteria or other combinations of clinical features could improve this disappointing figure. This second phase required the perspective of a clinical epidemiologist, and Andrew and I were indoctrinated with the novel concepts, at least for us, of sensitivity, specificity and positive and negative predictive values. ${ }^{3}$ These studies suggested there may well be a broader clinical phenotype to Parkinson's disease than was generally appreciated, and prompted a third study - a pathologico-clinical study of 100 cases of pathologically proven Parkinson's disease. ${ }^{4}$

Throughout the 1990s, many detailed clinic pathological studies were carried out on Parkinson's disease and atypical parkinsonian syndromes at the Queen Square Brain Bank and a decade later Andrew Hughes returned to do a sabbatical when he found gratifyingly that our pioneering work had led to a significant improvement in clinical accuracy in the diagnosis of Parkinson's disease in the UK. ${ }^{5} 6$

\section{Competing interests None.}

Provenance and peer review Commissioned; not externally peer reviewed.

Accepted 11 April 2012

Published Online First 29 May 2012

J Neurol Neurosurg Psychiatry 2012;83:954-955. doi:10.1136/jnnp-2012-302969

\section{REFERENCES}

1. Gibb WR, Lees AJ. The significance of the Lewy body in the diagnosis of idiopathic Parkinson's disease. Neuropathol Appl Neurobiol 1989;15:27-44.

2. Rajput AH, Rozdilsky B, Rajput A. Accuracy of clinical diagnosis in parkinsonism-a prospective study. Can J Neurol Sci 1991;18:275-8.

3. Hughes AJ, Ben-Shlomo Y, Daniel SE, et al. What features improve the accuracy of clinical diagnosis in Parkinson's disease: a clinicopathologic study. Neurology 1992;42:1142-6.

4. Hughes AJ, Daniel SE, Blankson S, et al. A clinicopathologic study of 100 cases of Parkinson's disease. Arch Neurol 1993;50:140-8.

5. Hughes AJ, Daniel SE, Ben-Shlomo Y, et al. The accuracy of diagnosis of parkinsonian syndromes in a specialist movement disorder service. Brain 2002:125:861-70.

6. Hughes AJ, Daniel SE, Lees AJ. Improved accuracy of clinical diagnosis of Lewy body Parkinson's disease. Neurology 2001:57:1497-9. 